\title{
Introductory notes for the Acta IMEKO third issue 2021 General Track
}

\author{
Francesco Lamonaca ${ }^{1}$ \\ ${ }^{1}$ Department of Computer Science, Modeling, Electronics and Systems Engineering (DIMES), University of Calabria, Ponte P. Bucci, 87036, \\ Arcavacata di Rende, Italy
}

\begin{abstract}
Section: EDITORIAL
Citation: Francesco Lamonaca, Introductory notes for the Acta IMEKO second issue 2021 General Track, Acta IMEKO, vol. 10, no. 3, article 3, September 2021, identifier: IMEKO-ACTA-10 (2021)-03-03
\end{abstract}

Received September 6, 2021; In final form September 9, 2021; Published September 2021

Copyright: This is an open-access article distributed under the terms of the Creative Commons Attribution 3.0 License, which permits unrestricted use, distribution, and reproduction in any medium, provided the original author and source are credited.

Corresponding author: Francesco Lamonaca, e-mail: f.lamonaca@dimes.unical.it

\section{INTRODUCTORY NOTES FOR THE ACTA IMEKO GENERAL TRACK}

The third issue 2021 of Acta IMEKO includes a General Track aimed at collecting contributions that do not relate to a specific event. As editor in chief, it is my pleasure to give readers an overview of these papers, with the aim of encouraging potential authors to consider sharing their research through Acta IMEKO.

Benedikt Seegerand and Thomas Bruns., in 'Primary calibration of mechanical sensors with digital output for dynamic applications', tackle the challenge of the dynamic calibration of modern sensors with integrated data sampling and purely digital output for the measurement of mechanical quantities, such as acceleration, angular velocity, force, pressure or torque. The proposed method is an extension of the established methods and devices that yields primary calibration results. The proposal focuses on primary accelerometer calibrations but can easily be transferred to other mechanical quantities.

In 'Analysis of the mathematical modelling of a static expansion system', Carlos Mauricio Villamizar Mora et al. analyse the suitability of different models to represent the final pressures in a static expansion system with two tanks. It is concluded that the use of the ideal gas model is adequate in most simulated conditions, while the assumption that the residual pressure is zero before expansion presents problems under certain conditions. An uncertainty analysis of the process is carried out, which leads to evidence of the high importance of uncertainty in a first expansion over subsequent expansion processes. Finally, an analysis of the expansion system based on uncertainty is carried out to estimate the effect of the metrological characteristics of the measurements of the input quantities. The proposed design process makes it possible to determine a set of restrictions on the uncertainties of the input quantities.
The transmission of medical data and the potential for distant healthcare structures to share experiments about a given medical case raise several conceptual and technical questions. Good remote healthcare monitoring involves more challenges with regard to personalised heath data processing than the traditional methods currently used in hospitals throughout the world. The adoption of telemedicine in the healthcare sector has significantly changed medical collaboration. However, to provide good telemedicine services through new technologies such as cloud computing, cloud storage and so on, a suitable and adaptable framework should be designed. Moreover, a secure and collaborative platform will enhance the decision-making process within the chain of medical information exchange, including between requesting agencies and physicians. In 'Collaborative systems for telemedicine diagnosis accuracy', Jacques Tene et al. provide an in-depth literature review on interactions between telemedicine and cloud-based computing. The paper further proposes a framework that can allow various research organisations, healthcare sectors and government agencies to log data, develop collaborative analysis and support decisionmaking. Case studies involving electrocardiograms and electroencephalograms demonstrate the benefit of the proposed approach in data reduction and high-fidelity signal processing at the local level, enabling the communication of extracted characteristic features to the cloud database.

In 'Uncertainty of factor $\mathrm{Z}$ in the gravimetric volume measurement', Mar Lar Win presents an interesting problem for the gravimetric volume measurement method. In this measurement, $\mathrm{Z}$ factors are generally used to balance the liquid volume, facilitating an easy conversion from the apparent mass. The uncertainty of measurement assigned to the measurement of a liquid volume can be divided into two uncertainty contributions: those components related to the mass measurements and those components related to the mass-tovolume conversion. However, uncertainty due to the $\mathrm{Z}$ factor is generally neglected in the uncertainty calculation of gravimetric 
volume measurement in some International Organization for Standardization standards and calibration guides. The paper describes the combined effects of the density of water, the density of reference weights and air buoyancy on the uncertainty of the $\mathrm{Z}$ factor and how this affects the uncertainty of the measurement result.

Most sensing networks rely on punctual/local sensors, thus lacking the ability to spatially resolve the quantity to be monitored (e.g. a temperature or humidity profile) without relying on the deployment of numerous inline sensors. Most quasi-distributed or distributed sensing technologies rely on the use of optical fibre systems; however, these are generally expensive, which limits large-scale adoption. Recently, elongated sensing elements have successfully been used with time-domain reflectometry (TDR) to implement diffused monitoring solutions. The advantage of TDR is that it is a relatively low-cost technology with adequate measurement accuracy and the potential to be customised to suit the specific needs of different application contexts of the 4.0 era.

Starting from these considerations, Cataldo et al., in the paper 'Microwave reflectometric systems and monitoring apparatus for diffused-sensing applications', addressees the design, implementation and experimental validation of a novel generation of elongated sensing element networks that can be permanently installed in the systems to be monitored and used to obtaining a diffused profile of the quantity to be monitored. In particular, three applications are considered as case studies: irrigation process monitoring in agriculture, leak detection in underground pipes and building structure monitoring.

Ding et al., in the paper 'The inhibition of biodegradation on building limestone by plasma etching' presents an innovative technique that has been recently applied in the cleaning of soiled archaeological objects. In the paper it is presented and discussed the use of low-pressure plasma etching in cleaning microbial contaminations on an oolitic limestone from an UNESCO World Heritage listed monument: the Batalha Monastery in Central Portugal. The cleaning effect was assessed by FTIR, SEM, optical microscope, and cell viability index measurement. The experimental results demonstrate that plasma etching can be regarded as a fast and eco-friendly conservation tool for stone heritage architecture.

This issue confirms that Acta IMEKO is the natural platform for disseminating measurement information and stimulating collaboration among researchers from many different fields who are united by their common interest in measurement science and technology.

Francesco Lamonaca

Editor in Chief 